\title{
Desarrollo Turístico versus Calidad y Sustentabilidad Percibidas: Barrancas del Cobre, México
}

\author{
Manuel Ramón González Herrera, PhD \\ Universidad Autónoma de Ciudad Juárez, México \\ Vinod Sasidharan, PhD \\ San Diego State University, California, US \\ Julián Alberto Álvarez Hernández, PhD \\ Universidad Autónoma de Ciudad Juárez, México
}

\section{Doi:10.19044/esj.2018.v14n35p1 ～URL:http://dx.doi.org/10.19044/esj.2018.v14n35p1}

\begin{abstract}
The study of perceptions about tourism development at the Copper Canyon Park in Chihuahua, Mexico, is presented in order to assess the perceived quality and sustainability of the current model of territorial tourism development. A qualitative methodology by means of an explanatory and deductive approach was used, through which valid qualitative data were obtained, such as words and texts based on personal opinions of the hosts and visitors, which were obtained through surveys, interviews and online opinion. The sample was composed of residents from Creel, Divisadero, Urique and Otero, as well as tourists who have visited the destination. As result, the characterization of the tourist development of Copper Canyon Park is presented, as well as the study of local opinion regarding the tourism development model, and the analysis of visitors' perceptions. It is concluded that the projection of tourism development using the opinions of hosts and visitors is a necessary tool for the participatory management of quality and territorial sustainability of tourism.
\end{abstract}

Keywords: Tourism, quality, sustainability, destination, Copper Canyon

\section{Resumen}

Se presenta el estudio de las percepciones sobre el desarrollo del turismo en el Parque Barrancas del Cobre, en Chihuahua, México, con el propósito de valorar la calidad percibida y la sustentabilidad relativas al modelo actual de desarrollo turístico territorial. Se utilizó una metodología cualitativa mediante un enfoque explicativo y deductivo, a través del cual se obtuvieron datos cualitativos válidos, como palabras y textos basados en las opiniones personales de los anfitriones y visitantes, los cuales se obtuvieron a 
través de encuestas, entrevistas y opiniones en línea; la muestra estuvo compuesta por residentes en Creel, Divisadero, Urique y Otero, así como por turistas que han visitado el destino. Como resultado se presenta la caracterización del desarrollo turístico de Parque Barrancas del Cobre, así como el estudio de la opinión local sobre el modelo de desarrollo turístico, y el análisis de las percepciones de los visitantes. Se concluye que la proyección del desarrollo turístico a partir de las opiniones de anfitriones y visitantes es una herramienta necesaria para la gestión participativa de la calidad y la sustentabilidad del turismo a nivel territorial.

Palabras claves: Turismo, calidad, sustentabilidad, destino, Cañón del Cobre

\section{Introducción}

La valoración del desarrollo turístico incorporando las perspectivas y opiniones de anfitriones y visitantes debe considerarse un requisito básico para la gestión participativa de la sustentabilidad del destino a nivel local (Clausen y Gyimóthy, 2016; Grybovych, 2012). Tal enfoque es particularmente necesario cuando los intereses de las partes involucradas se contraponen en la dimensión espacio-temporal; especialmente, en aquellos casos en que las expectativas de los turistas, la comunidad local y el contexto natural y/o cultural del destino no son "convergentes" (Crosby, 2015; Wang, et al, 2014).

Se asume como problema a resolver la necesidad de estudiar las dimensiones de calidad y sustentabilidad del desarrollo turístico a través de una investigación empírica, fundamentada en las opiniones y valoraciones directas o a través de sitios web (No y Kim, 2015) por actores sociales claves del destino. En línea con este planteamiento se generaron dos preguntas de investigación ¿Son convergentes las percepciones de la comunidad y los visitantes en términos de calidad y sustentabilidad del desarrollo turístico en las Barrancas del Cobre? ¿Cuál es el valor utilitario de la información proporcionada por la comunidad y los visitantes con respecto al modelo de desarrollo turístico actual?

En consecuencia, es objetivo general de la investigación estudiar las percepciones sobre el desarrollo turístico del Parque Barrancas del Cobre en Chihuahua, México, según opiniones de anfitriones y visitantes, con el fin de valorar la calidad percibida y la sustentabilidad del destino en el contexto del modelo actual de desarrollo turístico territorial.

Por ende, se espera contribuir a la comprensión y solución del problema mediante el análisis integrado del Parque Barrancas del Cobre como un espacio público de uso turístico susceptible de transformación sustentable. Tal propósito se fundamenta en el reconocimiento de las percepciones sobre el desarrollo del turismo de una manera holística, incorporando las opiniones de los actores en diferentes lugares del destino, así como las perspectivas de los 
visitantes; al tiempo que se posiciona el alto potencial que ofrecen las tecnologías de la información y la comunicación como plataforma para fomentar el uso de herramientas alternativas (Ali y Frew, 2014; Berné et al, 2015; Soteriades, 2012), asumiéndose que la búsqueda de la sustentabilidad solo es posible a través de la participación de todos los actores (Waligo, Clarke, y Hawkins, 2015; Zmyslony, 2014). Al incluir comunidades vulnerables específicas, los hallazgos proporcionan información $\mathrm{y}$ recomendaciones al gobierno y partes interesadas, enfatizando que las comunidades locales deben movilizarse y empoderarse para alcanzar un turismo sustentable.

Como antecedentes se valoraron estudios que han examinado la relación entre anfitriones y visitantes, demostrando que las actitudes de los residentes hacia los turistas y las conductas de los turistas en un destino están directamente influenciadas por los tipos de contactos e interacciones entre ambos grupos, la solidaridad y la distancia social entre los mismos (Dongoh et al, 2018; Tasci, 2009; Yilmaz y Tasci, 2013). Se constató además, que los modelos de gestión que consideran las opiniones y preferencias de las comunidades locales y de los visitantes con respecto al desarrollo turístico facilitan iniciativas para promover la participación comunitaria activa e integrada en los procesos de planificación y toma de decisiones (Guccio et al, 2017; Pulido-Fernández y Pulido-Fernández, 2017).

También se consideraron antecedentes relativos a las contradicciones identificadas entre el desarrollo turístico y los intereses de las comunidades indígenas en el destino, con énfasis en el paisaje natural y diversidad cultural (Valdivia, 2013; Mayorga, 2015). Tales contradicciones se han valorado críticamente como impactos negativos, tales como violación de los derechos indígenas, despojo territorial, afectaciones a sus lugares sagrados, pérdida de valor para el patrimonio natural y cultural, inadecuada integración de las construcciones a la arquitectura local, carencia de retribución de los beneficios para las comunidades indígenas, falta de consulta sobre proyectos de desarrollo turístico y procesos de apropiación del territorio, entre otros (Almanza, 2013; Almanza, 2014; Tierra Nativa, 2018). Estas problemáticas se han reportado en denuncias y estudios que se oponen a los proyectos mal conceptualizados e inadecuadamente planificados, $y$ han estado protagonizadas por los Rarámuri, asociaciones civiles e investigadores (Conferencia de Prensa, 2010; Quintana, 2012; Mayorga, 2015).

El artículo se compone de dos secciones que corresponden al estudio de la opinión pública local relativa al modelo de desarrollo del turismo implantado y al análisis de las percepciones de los visitantes con respecto al destino. Los principales desafíos superados incluyeron la falta de información turística sistematizada y oficial sobre la región, y la carencia de planes oficiales relacionados con el desarrollo del turismo local para consulta pública. 
Limitaron el estudio además, la inexistencia de una base de datos con información turística actualizada y de una Organización de Gestión de Destinos Turísticos; de igual forma, la falta de interés por parte de algunos actores demuestra restricciones para la planificación participativa del desarrollo turístico sustentable. Los hallazgos presentados se utilizarán para proyectar estudios a futuro con un alcance geográfico ampliado, incorporando a todas las comunidades locales y partes interesadas, lo cual que se llevará a cabo en coordinación con el gobierno local-regional y otras instituciones relacionadas.

\section{Enfoque teórico en el que se inserta la investigación}

En los últimos años diversos estudios científicos han intentado proporcionar una mejor comprensión y operacionalización de los conceptos de calidad y la sustentabilidad del desarrollo turístico (Cabrini, 2014), justificando la necesidad de "enfatizar el turismo sostenible como un fuerte paradigma y visión para el desarrollo alternativo" (Perona y Molina, 2016, p.527). En este contexto, el turismo comunitario surge como una opción sustentable para el desarrollo económico y social, junto a una distribución equilibrada de los beneficios a nivel local (Hamilton y Alexander, 2013; Perona y Molina, 2016). Este enfoque ha sido recientemente defendido como "una alternativa sostenible a los modelos de turismo masivo que tienen territorios mercantilizados, poblaciones humanas desplazadas $y$ han transformado sus espacios y condiciones de vida" (Palomino, Zamora y López, 2016, p.6).

Los Criterios Globales de Turismo Sostenible acentúan la necesidad de maximizar los beneficios sociales y económicos para la comunidad local y minimizar los impactos negativos para fomentar el desarrollo social comunitario, oportunidades de empleo, comercio justo, productos sostenibles, políticas contra el acoso y la explotación sexual, y la igualdad de oportunidades de empleo; reconociendo así que el turismo no debería afectar negativamente la calidad de vida de las comunidades locales (GSTC, 2012). Además, los criterios resaltan la necesidad de maximizar los beneficios para el patrimonio cultural y minimizar los impactos negativos, apoyar la adopción de códigos de conducta en sitios vulnerables, utilizar elementos artísticos locales, arquitectura o del patrimonio cultural, al tiempo que se garantiza que los derechos de propiedad intelectual de la comunidad sean respetados (GSTC, 2012). Las percepciones de estos criterios entre los diferentes grupos sociales que interactúan en el destino son indicadores factibles para examinar opiniones sobre la calidad y la sustentabilidad del turismo (Cárdenas, Byrd y Duffy, 2015).

Si bien la dimensión social se considera un componente importante del turismo sustentable, faltan todavía análisis adecuados de dicha dimensión 
debido a las complejidades derivadas de las interacciones que se dan un destino. En su investigación sobre el turismo en Iberoamérica González y Palafox (2014) exponen las insuficiencias que se presentan al evaluar la dimensión social del desarrollo del turismo sustentable; sin embargo, se ha demostrado que la participación ciudadana es una plataforma para profundizar la democracia a través de medios que enfaticen la justicia social, la dignidad humana y la solidaridad social (Rodríguez, 2015), así como la optimización de las relaciones entre la solidaridad emocional y la distancia social (Dongoh et al, 2018). Se reconoce además como base de este estudio que la sociedad comprometida "está directamente relacionada con la capacidad de hacer que la ciudadanía sea efectiva, y las entidades de iniciativa social jueguen un papel importante" (Rodríguez, 2015, p.13).

Al mismo tiempo, la ciudadanía cívica es considerada un "enfoque de empoderamiento decisivo para la participación comunitaria, ya que promueve la participación y la construcción de una sociedad civil, contribuyendo a generar respuestas creativas ante los problemas a través de los recursos existentes e impulsando los procesos de desarrollo y crecimiento personal" (Rodríguez, 2015, p.13). Por tanto, la participación de todos los actores involucrados a nivel del destino local constituye un marco apropiado para potencializar las sinergias requeridas en un espacio o territorio dado (Grybovych, 2012), incluyendo personas, grupos, instituciones públicas y privadas, programas, servicios, negocios, etc. (Rodríguez, 2015; Tuohino y Konu, 2014). Este enfoque se ha convertido en un importante factor de éxito para los proyectos turísticos a fin de facilitar la participación basada en la percepción de todos los implicados en la gestión del turismo (Postma, Cavagnarom y Spruyt, 2017), configurar la proyección deseada de la identidad local y determinar la imagen y el posicionamiento turístico deseado (Dioko, 2016).

A los fines del presente estudio, la percepción se conceptualiza como "el proceso a través del cual una persona selecciona, organiza e interpreta los estímulos para dar forma a una imagen significativa y coherente del mundo ... cómo vemos el mundo que nos rodea" (Schiffman, 2010, p. 157). En el ámbito de la gestión de destinos, la percepción es el proceso mediante el cual los visitantes y los anfitriones crean una imagen relacionada con el desarrollo del turismo. Tales percepciones también podrían interpretarse como impactos imaginarios o potenciales de los visitantes y los anfitriones con relación al desarrollo del turismo (Schiffman, 2010).

Con el fin de explicar el impacto de las experiencias de los visitantes sobre sus niveles de satisfacción de viaje, Suleyman et al, (2015) aporta la siguiente serie de constructos: competencia de comunicación intercultural, interacción cómoda, congruencia entre roles, percepción de distancia cultural, experiencias previas y satisfacción del visitante. Estos factores influyen 
directamente sobre las percepciones de los visitantes del destino y, principalmente, sobre las opiniones de calidad y sustentabilidad de conformidad con sus experiencias relativas al lugar de visita. De igual forma, el impacto de las experiencias previas de los anfitriones sobre el estado de su satisfacción es un factor determinante para la comprensión de este complejo fenómeno, proceso convergente en el que interactúan factores psicológicos, culturales, sociales y personales (Soria-Leiva, 2014, p.132).

\section{Marco metodológico}

Se utilizó una metodología cualitativa con enfoque explicativo y deductivo. Las estrategias para determinar la composición y tamaño de la muestra se centraron en obtener datos cualitativos válidos como palabras y textos basados en opiniones personales de los anfitriones y visitantes. La muestra se compuso por grupos de residentes locales y turistas que fueron seleccionados al azar, sin ser necesariamente representativos del universo o la población. Se implementó una estrategia de muestreo no probabilística por conveniencia o intencional, en la que los sujetos fueron seleccionados de acuerdo a su accesibilidad y proximidad al investigador. El estudio se contextualizó en los poblados de Creel, Divisadero y otros 9 poblados pequeños de la región, incluyendo además, a más de 60 pobladores dispersos que residen en las Barrancas de Urique y Otero (Ver fig.1); el trabajo de campo fue realizado durante el año 2016 y 2017.

Figura 1 Marco contextual de estudio

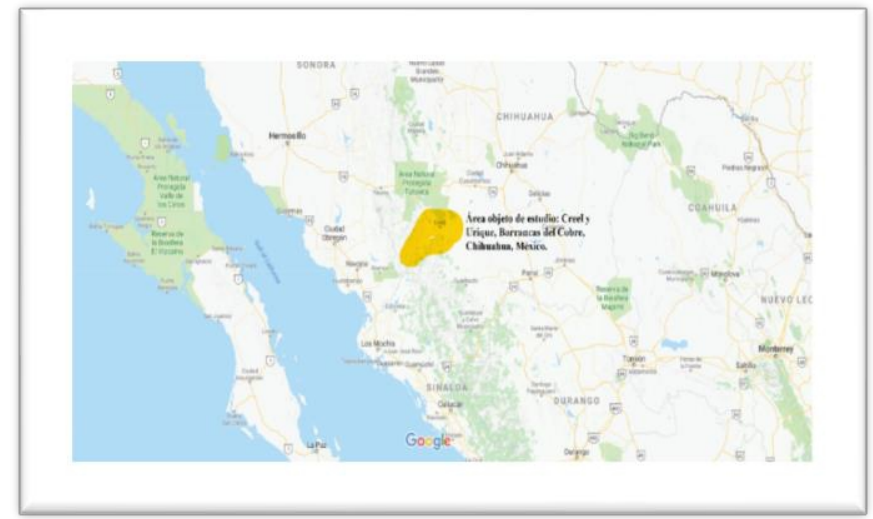

Fuente: elaboración propia basada en Map Data2018, Google INEGI

La implementación del estudio se basó en el Enfoque de Análisis Fenomenológico Interpretativo (Alase, 2017) para comprender las actitudes, opiniones, hábitos y motivaciones de las partes interesadas respecto a su participación y experiencias. Fueron implementadas varias técnicas para la recopilación, medición, análisis e interpretación de información como observación, encuestas con preguntas abiertas, entrevistas semiestructuradas, 
entrevistas grupales, consultas con especialistas, estudios de caso y grupos de discusión.

La exploración de las percepciones se realizó mediante entrevistas semiestructuradas y la administración de encuestas abiertas entre los residentes locales, en tanto la obtención de las opiniones de los visitantes respecto al destino utilizó diversas plataformas en línea tales como Expedia.com, Hotels.com y Tripadvisor.com. Los datos se obtuvieron a través de entrevistas con 12 especialistas del parque e instancias de turismo, 23 investigadores nacionales e internacionales consultados a través de redes sociales y de investigación, 11 académicos de las ciencias sociales con especialización en turismo, geografía, sociología, economía, administración y trabajo comunitario, así como 6 gerentes de 4 hoteles y 2 agencias de viajes locales. Además, se realizaron 328 encuestas con habitantes locales de diferentes localidades ( 6 en total), así como 185 consultas informales con residentes en espacios públicos de sus comunidades. También se recopiló información del sector privado del turismo a través de 16 pequeños grupos de debate con la participación de 3 a 5 personas en cada uno.

Para la puesta en práctica del estudio se identificaron cinco variables principales con el fin de conocer las experiencias y percepciones de la población anfitriona respecto al desarrollo del turismo, teniendo en cuenta los siguientes criterios: tipo de proyecto: percepciones de las comunidades anfitrionas sobre el proyecto turístico (opiniones, sugerencias, mejoras); necesidades y deseos de la comunidad: mejoras en la infraestructura urbana y turística del destino deseadas por la comunidad; implicación comunitaria: percepción de las comunidades anfitrionas sobre su rol y participación en las actividades turísticas locales; aspiraciones de la comunidad respecto al turismo: deseo de la comunidad de aprender lo importante que son para el desarrollo del turismo; y valor e importancia del desarrollo turístico local: percepciones de la comunidad local sobre los impactos del turismo.

El estudio de las percepciones de los visitantes se realizó mediante las opiniones en línea (Udayapuram y Gavirneni, 2015; Xiang, et al, 2017) disponibles en Expedia.com, Hotels.com y Tripadvisor.com; este análisis permitió conocer la valoración de los visitantes de Barrancas del Cobre de acuerdo a los criterios de evaluación de cada sitio web.

\section{Percepciones de las comunidades locales}

La realización del estudio se basó en la caracterización de las experiencias y satisfacción de los anfitriones con respecto al desarrollo del turismo local. Sobresale por su contribución a la investigación el aporte de la comunidad Creel - Pueblo Mágico-, ya que es el conjunto poblacional con mayor desarrollo y afluencia turística en la región, lo que determinó que los resultados obtenidos fueron los más significativos, representativos e 
integradores. El análisis realizado evidenció en términos generales que los pobladores no tienen suficiente información sobre el desarrollo turístico y desconocen los proyectos propuestos para convertir su espacio de residencia permanente en un Resort de Montaña internacional. Es evidente que si la población local no está informada, implicada y comprometida será poco probable alcanzar este fin; en particular, si los anfitriones no han sido educados en las dimensiones del turismo y la sustentabilidad no podrán ser conscientes y actuar a favor del desarrollo propuesto.

En base al análisis de los indicadores estudiados se verificó un alto nivel de insatisfacción de la comunidad con respecto al modelo de desarrollo del turismo territorial implantado. Al considerar los criterios establecidos por el GSTC, se advierte falta de planificación efectiva de la sustentabilidad con énfasis en el componente sociocultural, ya que no ha habido una maximización real de los beneficios sociales y económicos para la comunidad local; al tiempo que el turismo no ha contribuido a la reducción de los impactos negativos sobre el medio ambiente. Según las percepciones de los participantes es posible afirmar que se trata de un desarrollo que carece de vínculos y bases comunitarias sustentables, apartado de las opiniones de las comunidades anfitrionas que conocen bien el destino y carente de una adecuada orientación hacia el empoderamiento e implicación de los lugareños.

Atendiendo al diagnóstico de las necesidades sentidas por la comunidad, sus motivaciones, gustos y preferencias puede preverse que hacia escenarios futuros el estado de percepción de dichas comunidades locales podría afectar negativamente la percepción de los visitantes con respecto al desarrollo turístico regional, su imagen, arquitectura de marca y posicionamiento estratégico. Este escenario es previsible sobre todo en la medida en que el parque logre alcanzar los segmentos de mercado con mayores expectativas y experiencias relativas a este tipo de producto turístico; particularmente, aquellos interesados en las culturas auténticas y tradiciones conservadas, y que manifiestan altas exigencias para alcanzar una satisfacción favorable $\mathrm{y}$ experiencia memorable.

Varias preguntas realizadas a los participantes durante este estudio se enfocaron a comprender si la comunidad está realmente interesada en ser parte de las iniciativas de desarrollo turístico en Barrancas del Cobre. La mayoría de los actores consultados expresaron estar motivados y dispuestos a aprovechar esta oportunidad, al tiempo que reconocen la importancia del turismo para su bienestar, la conveniencia de ser capacitados como fuerza laboral para el turismo y ser tomados en consideración durante los procesos de planificación turística. En opinión de un residente local "el desarrollo del turismo aquí podría beneficiarnos, queremos integrarnos a este proyecto, queremos ser tomados en cuenta, pero este propósito lo hemos visto limitado hasta ahora ... no tengo la menor idea de cómo participar, pero quiero hacerlo... 
sé que es necesario hacer algo para recibir a cambio, así que estoy dispuesto a comenzar e integrarme, y dar lo mejor de mí".

\section{Percepciones de los visitantes con respecto al desarrollo del turismo}

Para el estudio de la opinión en línea se tomaron en consideración las opiniones de los visitantes en lo relativo al alojamiento, atractivos y actividades. Durante las etapas iniciales de la investigación se constató que los sitios web disponibles para la recopilación de opinión en línea sobre la satisfacción de los visitantes solo ofrecen posibilidades específicas y limitadas para calificar la calidad del producto y los servicios turísticos.

Las opciones de comentarios proporcionadas por los sitios web son indicadores generales de satisfacción del viaje, tales como limpieza y comodidad de las habitaciones, estado del hotel, servicio y personal de contacto, entre otros; así como atracciones naturales y parques, atracciones y lugares de interés, y actividades disponibles para los huéspedes. Estos sitios web no evalúan la sustentabilidad percibida del destino con relación al patrimonio local y a las características de la comunidad anfitriona. Sería recomendable que los sitios web brindaran opciones que permitieran comentarios de los visitantes respecto a las experiencias de sus contactos con la población local, la importancia y el estado de conservación del patrimonio, códigos de comportamiento de los visitantes, buenas prácticas ambientales, uso de productos locales, y bienestar económico de las comunidades. Esto favorecería una mayor sensibilidad con respecto a los aspectos de la sustentabilidad del destino y, en consecuencia, beneficiaría el posicionamiento del destino en los mercados de viajes sustentables.

Las respuestas de los visitantes que han estado en la Barranca de Urique fueron altamente significativas para la investigación, ya que esta área contiene la mayor concentración de infraestructura turística de las Barrancas del Cobre. Según información consultada en línea las evaluaciones realizadas por los visitantes fueron muy favorables, pero sus opciones de retroalimentación solo incluyeron aspectos relacionados con las instalaciones de alojamiento disponibles en el destino. Al respecto, un visitante mencionó "¡Un lugar que no te puedes perder, mucha adrenalina! ¡El parque es muy bueno! Las atracciones son las mejores ..., lo recomiendo todo. No es barato, pero vale la pena. El restaurante tiene buena comida y el precio es justo".

Según la opinión en línea dejada por los visitantes, se advierte que el destino es una excelente opción para descansar y apreciar la naturaleza; los comentarios aportan favorables niveles de percepción sobre los hoteles, destacando como relevante la calidad del descanso y el servicio. En la evaluación que realizaron otorgan al Hotel Misión la menor puntuación, lo cual resulta interesante ya que es el de mayor categoría; caso contrario ocurre con el Hotel Jade que ostenta menor categoría y recibe las mejores 
puntuaciones. Ninguna de las opiniones registradas relaciona el destino con las comunidades autóctonas; da la impresión que se genera una burbuja en la que los visitantes se aíslan de la población local, sobre todo de los indígenas; al tiempo que los sitios web de las propias empresas no dan opción directa para recibir comentarios al respecto, desvalorizándose así un atractivo de muy alta jerarquía.

El estudio prosigue con la valoración de las experiencias de los visitantes durante su estancia en el destino, en función de lo cual se consideraron cada una de las tres fracciones (término utilizado por la fuente): naturaleza y parques; tours \& actividades; y atracciones \& lugares de interés. La experiencia en la mayoría de los casos resulta muy positiva y rebasa las expectativas del visitante, lo cual es fundamentado en el sitio web Tripadvisor.

En este apartado del sitio web se relatan diversas reseñas positivas, al respecto los turistas califican la experiencia con adjetivos como "única, increíble e inolvidable", la gran mayoría califica los atractivos turísticos de la región como excelentes, y brindan comentarios mayoritariamente positivos. Tres de los ocho atractivos cuentan con certificado de excelencia otorgado por Tripadvisor, lo cual indica que constantemente han sido evaluados como atractivos de alta calidad. Cabe señalar que estas certificaciones se han puesto muy de "moda" entre los turistas potenciales como criterio para la toma de decisiones relativas a los viajes turísticos, por lo que muchas empresas los muestran orgullosamente junto a otros premios y certificaciones de calidad y sustentabilidad, además de su presencia habitual e influyente en diversos sitios de internet.

La mayoría de los turistas catalogan los tours y actividades ofrecidos en las Barrancas del Cobre como excelentes, narran como los servicios son de primera calidad y la experiencia que han vivido la describen como "maravillosa e inolvidable"; no obstante, una minoría califica como elevado el costo de El Chepe -tren turístico-. Muchos de los atractivos culturales visitados en el destino corresponden a misiones y lugares religiosos, por lo que los turistas califican el lugar como "mágico", "diferente" y "silencioso". Mayormente describen y califican la experiencia como "inusual" y "única", además destacan las caminatas que se realizan para llegar a los atractivos como "memorables". El estudio realizado permitió advertir que las opiniones ofrecidas por los visitantes suponen una ecuación de calidad en la que las expectativas son inferiores a la experiencia vivida; la calidad es percibida como buena y muy buena; la satisfacción es alta y muy alta; lo cual favorece la intención futura de recomendar favorablemente y volver a visitar el lugar.

Los visitantes mayoritariamente califican el parque de excelente, dicha categoría representa el $84 \%$ del total de las evaluaciones, razón por la cual cuenta con un Certificado de Excelencia otorgado por Tripadvisor. Esto significa que el parque se encuentra evaluado con puntuaciones muy altas; 
además, la página web mencionada lo recomienda como la opción número uno en Chihuahua para realizar actividades al aire libre, lo cual revela que el destino cubre las expectativas del visitante, además de generar experiencias perdurables.

Como resultado del estudio de percepción realizado se comprobó que las atracciones del parque son identificadas como su mayor fortaleza; aunque se reconocen también la belleza del paisaje, y la excelente preparación y atención de los empleados del parque. Sobresalen entre los comentarios más positivos el recorrido a través de la tirolesa más larga del mundo y el paseo en el teleférico, describiendo la experiencia como "inolvidable", "extrema", e "increíble". Los visitantes indican que el precio del parque es algo elevado, que la publicidad que se le hace a este y a sus paquetes es escasa, y que la información turística es insuficiente. En algunos casos se menciona la falta de mobiliario, como casilleros para resguardar las pertenencias al momento de realizar los recorridos.

\section{Conclusión}

La nueva base de conocimientos turísticos proporcionada por el presente estudio contiene información relevante relativa a las percepciones de los visitantes y anfitriones con respecto al modelo de desarrollo turístico local en términos de calidad y sustentabilidad territorial, mostrando similitudes y divergencias entre las preocupaciones de la comunidad y los visitantes con respecto a los impactos del turismo. Se corroboró el valor metodológico de la integración de las dos variables utilizadas, enfoque este que podría aplicarse en escenarios futuros para facilitar un mejor proceso de toma de decisiones orientado a la co-creación (Lin, Chen y Filieri, 2017) de un modelo de desarrollo de turismo sustentable (GSTC, 2012; Peric y Djurkin, 2014) en este destino de montaña.

El estudio comprobó que las percepciones de la comunidad local y de los visitantes hacia el desarrollo del turismo en Barrancas del Cobre no son convergentes en términos de calidad y sustentabilidad, lo cual se contrapone a lo que diferentes autores han demostrado debería ser (Palafox, Pineda, y Anaya, 2013; Palomino, Zamora, y López, 2016; Peric y Djurkin, 2014; Postma, Cavagnaro, y Spruyt, 2017); además, las comunidades indígenas no son percibidas por los visitantes como una atracción de alta jerarquía para el destino. Es necesario desarrollar estrategias a nivel local como otros destinos ya han adoptado (Ali y Frew, 2014; Bhat y Gaur, 2012; Bimonte y Punzo, 2016; Pulido-Fernández y Pulido-Fernández, 2017) para asegurar un nexo satisfactorio de intereses y motivaciones entre anfitriones y visitantes (Byrd, 2007; Crosby, 2015; Grybovych, 2012) y garantizar así la creación de experiencias de viaje memorables dentro del destino. Mediante este enfoque se puede avanzar hacia el desarrollo de un sistema de gestión turística eficiente 
e integrado que consolide la inteligencia de mercado, la sustentabilidad, la participación de los actores locales, la competitividad empresarial y la rentabilidad del destino turístico.

Hasta el presente la atracción turística hacia el Parque Barrancas del Cobre se ha concentrado apenas en la topografía accidentada y su paisaje natural. Desde una perspectiva de sustentabilidad social es esencial integrar las opiniones de los residentes y visitantes dentro de la planificación turística de una manera más compatible (Clausen y Gyimóthy, 2016; Guccio et al, 2017; Suleyman et al, 2015), evitando así la propagación de un modelo de turismo masivo que excluya los intereses de la comunidad. Ello podría impedir la sobre comercialización del territorio y una transformación del espacio natural y la cultura local al estilo de los parques de aventura más convencionales. Desde una perspectiva comercial tales actuaciones podrían crear una identidad de base local para el destino y permitir ajustes que satisfagan las demandas del creciente segmento de mercado de viajes sustentables, las cuales están orientadas hacia nuevos productos turísticos de alto valor en términos integrados de calidad y sustentabilidad.

La información proporcionada por la comunidad local y los visitantes fue de alto valor utilitario para comprender los problemas asociados a la valoración de la dimensión social del desarrollo del turismo sustentable a nivel de destino, lo que también ha sido demostrado en investigaciones anteriores (Dongoh et al, 2018; Montaño, Núñez, y Pérez, 2016; Wang, et al, 2014). El estudio de las comunidades locales brindó la oportunidad de escuchar, observar y comprender las percepciones de la población, demostrando el alto valor de la experiencia de los actores sociales para la implementación de modelos de desarrollo turístico a nivel local. Simultáneamente, las valoraciones en línea de los visitantes agregaron información valiosa, corroborando su aplicabilidad como una nueva herramienta para evaluar las experiencias de los visitantes (Udayapuram y Gavirneni, 2015), esto permitió a los investigadores concluir que tales fuentes tienen un gran potencial de uso si se manejan como herramientas avanzadas para la gestión inteligente de un destino, centrándose en la calidad y la sustentabilidad.

Se reveló que el apego de los residentes locales hacia la comunidad y su participación en los procesos de gestión a nivel de destino son factores clave que afectan los niveles de apoyo necesarios para el desarrollo del turismo sustentable en Barrancas del Cobre, demostrado que hay mayores oportunidades de éxito en los destinos donde las opiniones de las partes interesadas se toman en consideración durante el proceso de planificación y gestión del destino (Tuohino y Konu, 2014). Se hizo evidente que cuando el modelo de turismo local no es endógeno, participativo y autogestionado los habitantes no perciben los beneficios; por tanto, se generan incompatibilidades que desfavorecen la sustentabilidad. Este problema se evidenció a través de 
los impactos ambientales y socioculturales adversos generados por el desarrollo del turismo en Barrancas del Cobre, los cuales deberán corregirse para promover un modelo de turismo alternativo local.

El acceso a las comunidades de Barrancas del Cobre estuvo restringido debido a la ubicación geográfica y el relieve montañoso de esta región; por tanto, este estudio incluyó solo los territorios accesibles, más vulnerables e importantes afectados por el desarrollo del turismo. Por ello, el número de participantes, así como las características específicas de los sujetos pudieran haber limitado la confianza de los hallazgos y su aplicabilidad a otras poblaciones de contexto similar. Otra limitación de este estudio fue que el análisis de la percepción integrada del destino no incorporó la totalidad de las comunidades locales y barrancas con atractivos turísticos, razón por la que es recomendable que se realice un estudio a mayor escala como continuidad de la investigación. Tal estudio sería de gran valor, ya que a pesar de que las localidades de las Barrancas del Cobre tienen características similares y comparten el mismo espacio geográfico, los contextos para el desarrollo turístico difieren de un lugar a otro; además, la obtención de opiniones sobre el desarrollo del turismo por parte de una mayor cantidad de anfitriones y visitantes es esencial para un enfoque integrado en la Gestión del Destino Barrancas del Cobre.

\section{References:}

1. Alase, A. (2017). The Interpretative Phenomenological Analysis (IPA): A guide to a good qualitative research approach. International Journal of Education \& Literacy Studies, 5(2). http://dx.doi.org/10.7575/aiac.ijels.v.5n.2p.9.

2. Ali, A., y Frew, A. J. (2014). Technology innovation and applications in sustainable destination development. Information Technology \& Tourism, 14(4), 265-290. https://doiorg.libproxy.sdsu.edu/10.1007/s40558-014-0015-7.

3. Almanza Alcalde, Horacio. (2013). El Proyecto Barrancas del Cobre en Chihuahua y la Violación a los Derechos Indígenas. Ponencia presentada en el VIII Congreso de Investigadores INAH "El INAH en la Modernidad" Atlixco Puebla del 19 al 22 de noviembre 2013. https://www.academia.edu/5328846/El_Proyecto_Barrancas_del_Co bre_en_Chihuahua_y_la_Violaci\%C3\%B3n_a_los_Derechos_Ind\%C $3 \%$ ADgenas

4. Almanza Alcalde, Horacio. (2014). Paradojas del Turismo: entre la transformación y el despojo. Los Casos de Mogotavo y Wetosachi, Chihuahua, México. Revista de Análisis Turístico, $\mathrm{n}^{\circ} 18,2^{\circ}$ semestre 2014, pp. 45-56 file://C:/Users/Lenovo/Downloads/186-679-2PB.pdf 
5. Berné, C., García-González, M., García-Uceda, M. E., y Múgica, J. M. (2015). The effect of ICT on relationship enhancement and performance in tourism channels. Tourism Management, 48(2015), 188-198. https://doi.org/10.1016/j.tourman.2014.04.012.

6. Bhat, S., y Gaur, S. S. (2012). Managing diverse stakeholders in the context of destination marketing. Worldwide Hospitality and Tourism Themes, 4(2), 185-202. https://doi.org/10.1108/17554211211217352.

7. Bimonte, S., y Punzo, L. F. (2016). Tourist development and hostguest interaction: An economic exchange theory. Annals of Tourism Research, 58(2016),

128-139. https://doi.org/10.1016/j.annals.2016.03.004.

8. Byrd, E. T. (2007). Stakeholders in sustainable tourism development and their roles: Applying stakeholder theory to sustainable tourism development. Tourism Review, 62(2), 6-13. https://doi.org/10.1108/16605370780000309.

9. Cabrini, L. (2014). Trends in sustainable tourism and the role of the Global Council (GSTC). First Congress of Energy Efficiency and Sustainability in the Tourism Sector. Palma de Mallorca, Spain. Accessed: 06/02/2016 from http://docplayer.es/2312294-Tendenciasdel-turismo-sostenible-y-el-rol-del-consejo-global-gstc.html.

10. Cárdenas, D. A., Byrd, E. T., y Duffy, L. N. (2015). An exploratory study of community awareness of impacts and agreement to sustainable tourism development principles. Tourism and Hospitality Research, 15(4), 254-266. https://doiorg.libproxy.sdsu.edu/10.1177/1467358415580359.

11. Clausen, H. B., y Gyimóthy, S. (2016). Seizing community participation in sustainable development: Pueblos Mágicos of Mexico. Journal of Cleaner Production, $111 B$ (2016), 318-326. https://doi.org/10.1016/j.jclepro.2015.01.084.

12. Conferencia de Prensa. (2010). Comunidades indígenas denuncian proyecto turístico en Barrancas del Cobre, Chihuahua. Boletín de Prensa Chihuahua, Chihuahua, México, Publicado 11 de agosto de 2010. https://ewwaunel.wordpress.com/2010/08/11/comunidadesindigenas-denuncian-proyecto-turistico-en-barrancas-del-cobrechihuahua/

13. Crosby, A. (2015). Convergence of interests between tourists, locals and territory. Naturalae. Accessed: 06/07/2016 from http://www.efeverde.com/opinion/convergencia-de-intereses-entreturistas-locales-y-las-areas-naturales.

14. Dioko, L. A. N. (2016). Progress and trends in destination branding and marketing: A brief and broad review. International Journal of 
Culture, Tourism and Hospitality Research, 10(1), 5-13. https://doi.org/10.1108/IJCTHR-12-2015-0145.

15. Dongoh, J., Tasci, D. A., Woosnam, K. M., Maruyama, N. U., Hollas, Cr. R., y Aleshinloye, K. D. (2018). Residents' attitude towards domestic tourists explained by contact, emotional solidarity and social distance. Tourism Management, 64, 245-257. https://doi.org/10.1016/j.tourman.2017.08.012.

16. Global Sustainable Tourism Council (GSTC). (2012). Global sustainable tourism criteria for hotels and tour operators, version 2, 23 February 2012, Madrid, Spain. Accessed: 05/06/2016 from https://www.gstcouncil.org/en/gstc-criteria/criteria-for-hotels-andtour-operators.html.

17. González, D., y Palafox, A. (2014). Sociology of tourism in Spanish: Exploratory review of articles published in Ibero-American journals 2003-2013. Studies and Perspectives in Tourism, 23(2014), 805-819.

18. Grybovych, O. (2012). Designing a qualitative multi-case research study to examine participatory community tourism planning practices. Advances in Culture, Tourism and Hospitality Research, 6, 501-520. Emerald Group Publishing Limited.

19. Guccio, C., Lisi, D., Martorana, M., y Mignosa, A. (2017). On the role of cultural participation in tourism destination performance: an assessment using robust conditional efficiency approach. Journal of Cultural Economics, 41(2), 129-154. https://doiorg.libproxy.sdsu.edu/10.1007/s10824-017-9295-z.

20. Hamilton, K., y Alexander, M. (2013). Organic community tourism: A co-created approach. Annals of Tourism Research, 42(2013), 169-190. https://doi.org/10.1016/j.annals.2013.01.015.

21. Lin, Z., Chen, Y., y Filieri, R. (2017). Resident-tourist value cocreation: The role of residents' perceived tourism impacts and life satisfaction. Tourism Management, 61(2017), 436-442. https://doi.org/10.1016/j.tourman.2017.02.013.

22. Mayorga, Patricia. (2015). Rarámuri se oponen a proyectos depredadores en Barrancas del Cobre. Proceso.com.mx. 27 de mayo de 2015. https://www.proceso.com.mx/405526/raramuris-se-oponena-proyectos-depredadores-en-barrancas-del-cobre

23. Montaño, A., Núñez, G., y Pérez, J. (2016). Threat and challenges of Los Cabos as a consolidated destination: the perception of local entrepreneurs. Economy and Politics Magazine, 22, 35-43. Accessed: $05 / 12 / 2016$ from http://www.ucuenca.ec/ojs/index.php/REP/article/view/811. 
24. No, E., y Kim, J. K. (2015). Comparing the attributes of online tourism information sources. Computers in Human Behavior, 50(2015), 564 575. https://doi.org/10.1016/j.chb.2015.02.063.

25. Palafox, A., Pineda, G., y Anaya, J. (2013). Resident`s perceptions in a tourist destination. In A. A. Herrera \& A. G. Damián (Eds.), Sustainable tourism management. Conceptual base and case studies (pp. 121-142). Cozumel, Mexico: UQROO.

26. Palomino, B., Zamora, J., y López, G. (2016). Community tourism in the Sierra Norte de Oaxaca: Perspective from institutions and governance in indigenous territories. The Sustainable Periplo, 30, 637. Accessed: 05/2/2016 from http://rperiplo.uaemex.mx.

27. Peric, M., y Djurkin, J. (2014). Systems thinking and alternative business model for responsible tourist destination. Kybernetes, 43(3/4), 480-496. https://doi.org/10.1108/K-07-2013-0132.

28. Perona, E., y Molina, E. (2016). Evolution and stages of tourism development in the province of Córdoba, Argentina: How far is the sustainable tourism ideal? Notebooks of Tourism, 37, 527-530.

29. Postma, A., Cavagnaro, E., y Spruyt, E. (2017). Sustainable tourism 2040. Journal of Tourism Futures, 3(1), 13-22. https://doi.org/10.1108/JTF-10-2015-0046.

30. Pulido-Fernández, J. I., y Pulido-Fernández, M. C. (2017). Proposal for an indicators system of tourism governance at tourism destination level. Social Indicators Research, 1-49. https://doiorg.libproxy.sdsu.edu/10.1007/s11205-017-1627-z.

31. Quintana S., Víctor M. (2012). Dignidad Rarámuri en Acción. La Jornada. Viernes 28 de septiembre de 2012. https://www.jornada.com.mx/2012/09/28/opinion/020a2pol

32. Rodríguez, M. (2015). The praxis of community social work at the local level: citizenship, participation, empowerment. AIS, Actions and Social Investigations, 35, 7-29.

33. Schiffman, L. (2010). Consumer behavior. Tenth edition. Mexico: Pearson Education.

34. Soria-Leyva, E. (2014). Obtaining a fuzzy indicator to quantify the impacts generated by tourism in receiving communities case study. Yearbook of Faculty of Economics and Business Sciences (AFCEE), 5, 131-148. Accessed: 05/07/2016 from http://ojs.uo.edu.cu/index.php/aeco/article/view/555/530.

35. Suleyman, N., Ihtiyar, A., Shah, F., y Faizan, A. (2015). A conceptual framework to explain the impact of visitors' previous experiences on customer satisfaction. International Interdisciplinary BusinessEconomics Advancement Journal, 1(1), 38-49. 
36. Tasci, D. A. (2009). Social distance: The missing link in the loop of movies, destination image, and tourist behavior? Journal of Travel Research, 47(4), 494-507. https://doiorg.libproxy.sdsu.edu/10.1177/0047287508326534.

37. Tierra Nativa. (2018). Los 10 bárbaros del turismo en la Barranca de Cobre. Tierra Nativa A.C. Asociación Civil No Lucrativa en México. http://www.tierranativa.org/index.php?IDDT $=181 \& O P T 2=154 \& N I V$ EL1

38. Tuohino, A., y Konu, H. (2014). Local stakeholders' views about destination management: Who are leading tourism development? Tourism Review, 69(3), 202-215. https://doi.org/10.1108/TR-062013-0033.

39. Udayapuram, H. P., y Gavirneni, S. (2015). Analyzing online reviews: New tools for evaluating visitor experiences. Research \& Policy Brief Series, 67. Ithaca, NY: Community and Regional Development Institute, Cornell University. https://cardi.cals.cornell.edu/publications/research-policybriefs/analyzing-online-reviews-new-tools-evaluating-visitorexperiences.

40. Valdivia Ramírez, Fátima del Rocío. (2013). Las Barrancas del Cobre en la Sierra Tarahumara Contradicciones del reconocimiento de la Diversidad Cultural en un contexto neoliberal. Tesis para Optar al Grado de Maestra en Antropología. Centro de Investigaciones y Estudios Superiores en Antropología Social. Oaxaca, México. https://docplayer.es/74652179-Las-barrancas-del-cobre-en-la-sierratarahumara-contradicciones-del-reconocimiento-de-la-diversidadcultural-en-un-contexto-neoliberal-t-e-s-i-s.html

41. Waligo, V., Clarke, J., y Hawkins, R. (2015). Embedding stakeholders in sustainable tourism strategies. Annals of Tourism Research, 55(2015), 90-93. https://doi.org/10.1016/j.annals.2015.09.002.

42. Wang, S., Zhou, L., Lee, S., y King, C. (2014). Analysis of residents' social identity, tourism engagement, and propensity for tourism advocacy. Advances in Hospitality and Leisure, 10, 109-129. Emerald Group Publishing Limited.

43. Xiang, Z., Du, Q., Ma, Y., y Fan, W. (2017). A comparative analysis of major online review platforms: Implications for social media analytics in hospitality and tourism. Tourism Management, 58(2017), Pages 51-65. https://doi.org/10.1016/j.tourman.2016.10.001.

44. Zmys̀lony, P. (2014). Identification of leadership in emerging tourist destinations. Tourism Review, 69(3), 173-186. https://doi.org/10.1108/TR-06-2013-0031. 